\title{
grc $\nu \nu \gamma$ : Event generator for the single- and double-photon emission associated with neutrino pair-production
}

\author{
Y. Kurihara*, J. Fujimotoł T. Ishikawa‡ Y. Shimizu ${ }^{\S}$ \\ High Energy Accelerator Research Organization(KEK), \\ Tsukuba, Ibaraki 305-0801, Japan \\ T. Munehisa \\ Yamanashi University, Kofu, Yamanashi 400-8510, Japan
}

\begin{abstract}
A new event generator is proposed for two processes $e^{+} e^{-} \rightarrow \nu \bar{\nu} \gamma$ and $e^{+} e^{-} \rightarrow \nu \bar{\nu} \gamma \gamma$ where $\nu \bar{\nu}$ includes all the neutrino species. The exact matrix elements of single- and double-photon emission, generated by the GRACE system, are convoluted with the QED parton shower(QEDPS) to deal with the initial state radiations(ISR). It is pointed out that a careful treatment is required to avoid the double counting of the radiative photons between the matrix elements and the ISR part. A detailed comparison of grc $\nu \nu \gamma$ with the $\mathcal{O}(\alpha)$ calculations and other similar Monte Carlo generators is discussed on the total cross section and on various distributions. It is also examined how the possible effects of the anomalous triple-gauge-boson couplings can be observed.
\end{abstract}

*yoshimasa.kurihara@kek.jp

†junpei.fujimoto@kek.jp

†tadashi.ishikawa@kek.jp

$\S$ yoshimitzu.shimizu@kek.jp

『munehisa@hep.esb.yamanashi.ac.jp 


\section{Introduction}

The processes of single- and double-photon emission associated with a large missing energy are very important for the precision test of the standard model and the search for any new (invisible) particles beyond the standard model. In fact the number of the light-neutrino generations has been measured using this process on the $Z$-pole at the LEP experiments [1]. The efforts to find new invisible particles, such as a neutral (lightest) SUSY particle or a sequential heavy neutrino, are now continued by the LEP-2 experiments at higher energies[2]. In this case, however, the neutrino pair-productions with hard-photon emission are the main background for the investigation. With the increase of the accumulated luminosity, the cross sections of these processes must require more precision with an uncertainty of $\mathcal{O}(1 \%)$. In addition since $e^{+} e^{-} \rightarrow \nu_{e} \bar{\nu}_{e} \gamma(\gamma)$ includes the triple(quartic) gauge couplings, there is a possibility to find some new effects beyond the standard model on these rather poor-tested couplings.

There are some methods to estimate the cross sections including the higher order QED corrections. The simplest one would be to combine the matrix elements of $e^{+} e^{-} \rightarrow \nu \bar{\nu}$ with some tool for the initial-state radiations(ISR). The hard photon(s) may be supplied, for instance, by a parton shower, which can treat the photons with a finite transverse momentum $\left(p_{T}\right)$. When this method is applied to the center-of-mass system(CMS) energies above the $Z$-boson mass, however, the leading logarithmic (LL) approximation is not sufficient. This is because the colliding energy after the photon emission can match the $Z$-pole('Radiative Return'), and the probability to emit high-energy photon(s) is largely enhanced. When this happens the LL approximation is not successful in describing the spectrum around the $Z$ pole. To improve the precision of the calculations the non-LL terms for ISR must be taken into account as well as the LL terms. However, there exist no complete calculations up to the non-LL order.

Another possible way is to make use of the matrix elements of $e^{+} e^{-} \rightarrow$ $\nu \bar{\nu} \gamma(\gamma)$ 円 together with the ISR tool. Diagrams of the single(double)-photon emission are shown in Fig.1 (Fig.2), respectively. The visible and invisible hard-photon(s) are fed by the matrix elements as well as by the ISR tools.

${ }^{1}$ The complete matrix elements of the $e^{+} e^{-} \rightarrow \nu_{\mu} \overline{\nu_{\mu}} \gamma$ process and their compact approximated form were first given in Ref.[3]. 
Here it is crucial to avoid the double counting in the radiations coming from these two different sources. The conventional QED structure function is not suitable for this purpose because it is obtained after the integration over all allowed phase-space of the emitted photons except for their energy. Thus the double counting necessarily happens and cannot be excluded.

There exists no full electroweak one-loop calculation for $e^{+} e^{-} \rightarrow \nu \bar{\nu} \gamma$. Only the limited corrections, one-loop QED corrections and self-energy corrections to the $Z$-exchange diagrams[3, 4, 5] are available. Though there is no one-loop calculation at all for the $\nu_{e} \bar{\nu}_{e} \gamma$ process, the ISR tools might cover the most important corrections and allows us to give a realistic event generator which can meet the required theoretical uncertainty of $\mathcal{O}(1 \%)$.

The KORALZ Monte Carlo program [6] exploits the exact matrix elements of the single-photon processes with $\nu_{\mu}$ and $\nu_{\tau}$ production(an approximation is made for $\nu_{e}$ ) together with the exclusive exponentiation of YFS [7] as the ISR tool. Another event generator is NUNUGVP [8], which uses the exact matrix elements of the single- and multi(up to three)-photon emission(for all kinds of neutrinos) generated by the ALPHA algorithm 9] and the $p_{T^{-}}$ depending structure function [10] for ISR. Both programs include anomalous triple-gauge-boson coupling(TGC) in the package.

In this report a new event generator grc $\nu \nu \gamma$ is proposed. It adopts the exact matrix elements for $e^{+} e^{-} \rightarrow \nu \bar{\nu} \gamma(\gamma)$ generated by means of the GRACE system 11] combined with QEDPS 12] for ISR. The advantages of these packages are:

- The exact matrix elements up to the double-photon emission, including the $\nu_{e}$ process, are used. Double-photon emission is practically sufficient for experimental analysis.

- QEDPS keeps the complete kinematics for the emitted photons and virtual electrons before collisions. It allows a more flexible treatment of the ISR effects in avoiding the double-counting.

In section 2, the calculation method, particularly how to connect QEDPS to the radiative processes without double counting, is explained. The numerical results of single- and double-photon emission are presented in section 3. A detailed comparison among grc $\nu \nu \gamma$, the exact $\mathcal{O}(\alpha)$ calculations, KORALZ and NUNUGPV is also given in this section. The effects of the anomalous TGC in the single-photon events are discussed in section 4 . The conclusions are 
summarized in section 5. Appendix describes how to obtain and use the grc $\nu \nu \gamma$ program.

\section{Calculation Method}

The exact matrix-elements of all the processes are produced by an automatic calculation system GRACE. It generates the FORTRAN routines needed to calculate the amplitudes numerically based on the helicity-amplitude formalism.

For ISR the parton shower algorithm for QED, QEDPS, is used. In this algorithm the Altarelli-Parisi equation is solved in the LL approximation I4 using the Monte Carlo method. The details of this method can be found in Ref. 12. Here we recall that the algorithm can maintain the exact kinematics during the evolution of an electron. Let us consider the branching process

$$
e^{-}\left(x, K^{2}\right) \rightarrow e^{-}\left(x y, K_{1}^{2}\right)+\gamma\left(x(1-y), Q_{0}^{2}\right),
$$

where the parent electron, whose virtuality is $K^{2}$, has the momentum $p=$ $\left(E, \mathbf{0}_{T}, p_{z}\right), E=\sqrt{p_{z}^{2}-K^{2}}$ and $p_{z}=x p^{*}$ with $p^{*}$ being the momentum of the system in the infinite-momentum-frame. A cutoff mass $Q_{0}$ is introduced for the photon. Then the momenta of the daughter particles, $p_{1}$ for $e^{-}$and $p_{2}$ for $\gamma$, can be expressed as

$$
\begin{aligned}
& p_{1}=\left(E_{1}, \mathbf{k}_{T}, y p_{z}\right) \\
& p_{2}=\left(E_{2},-\mathbf{k}_{T},(1-y) p_{z}\right)
\end{aligned}
$$

where

$$
\begin{aligned}
& E_{1}=\sqrt{y^{2} p_{z}^{2}+\mathbf{k}_{T}^{2}-K_{1}^{2}}, \\
& E_{2}=\sqrt{(1-y)^{2} p_{z}^{2}+\mathbf{k}_{T}^{2}+Q_{0}^{2}} .
\end{aligned}
$$

Assuming $p^{*} \rightarrow \infty$, we have

$$
-K^{2}=-K_{1}^{2} / y+Q_{0}^{2} /(1-y)+\mathbf{k}_{T}^{2} /(y(1-y)),
$$

which determines $\mathbf{k}_{T}^{2}$ from $y, K^{2}$ and $K_{1}^{2}$. Hence one can obtain the distribution of the transverse momentum of the emitted photons, $\mathbf{k}_{T}^{2}$. Moreover this 
enables us to find the virtuality of the electron, $-K^{2}$, as well as its energy, just before the collision.

The phase-space integration of the matrix element squared is carried out numerically by BASES 15 using an adaptive Monte Carlo method. The integration is done in the five(eight)-dimensional kinematical phase-space of the three(four)-body final state. The evolution of the initial-state electrons by QEDPS is treated independently of BASES.

Since the QEDPS can provide a complete kinematical information about the emitted photons and the virtual electrons, it is easy to distinguish the photons from the matrix-elements and those from the parton shower. Let us divide the full one-photon phase space $\Omega_{\gamma}$ into two regions: One is for the visible region, $\Omega_{\mathrm{v}}$, and the other is for the invisible region, $\Omega_{\mathrm{i}}$. These are defined as

$$
\begin{aligned}
& \Omega_{\mathrm{v}}=\left\{\left(E_{\gamma}, \theta_{\gamma}\right) \mid E_{\gamma}>E_{0}, \theta_{0}<\theta_{\gamma}<180-\theta_{0}\right\}, \\
& \Omega_{\mathrm{i}}=\Omega_{\gamma}-\Omega_{\mathrm{v}},
\end{aligned}
$$

where $E_{\gamma}$ is the energy of the photon and $\theta_{\gamma}$ is its polar angle in degree from the beam axis. The minimum energy and angle, $E_{0}$ and $\theta_{0}$, must be given by the experimental condition. First the photons from QEDPS, $\gamma_{\mathrm{ps}}$ 's, are restricted to go inside of $\Omega_{i}$ and the photon from the matrix-elements, $\gamma_{\mathrm{ME}}$, is emitted into $\Omega_{\mathrm{v}}$. Since there is no overlap between these two regions no double-counting occurs. In addition the ordering of the electron virtuality is also required. During the evolution of an electron the virtuality is monotonically increasing, which is realized naturally in the QEDPS algorithm. A further condition must be imposed on the virtuality of the electron in the matrix-elements after emitting the photon: It should be greater than the virtuality of the electron in the last stage of QEDPS.

The configuration mentioned here cannot cover all the configurations for the photon emission. When $n$ photons are emitted from one electron line, the first $(n-1)$ photons are fed by QEDPS, including the statistical factor of $1 /(n-1)$ !, and only the last photon is supplied by the matrix element. This configuration represents the case where the first $(n-1) \gamma_{\mathrm{ps}}$ 's are invisible but only the last one, $\gamma_{\mathrm{ME}}$, is visible. Another case also exists. One of the first $(n-1) \gamma_{\mathrm{ps}}$ 's goes into $\Omega_{\mathrm{v}}$ while the other $(n-2) \gamma_{\mathrm{ps}}$ 's and $\gamma_{\mathrm{ME}}$ escape into $\Omega_{i}$. Though $\gamma_{\mathrm{ME}}$ is invisible in this case, the results are free from any infrared divergence thanks to the virtuality cut $Q_{0}$ introduced above. The 
minimum energy of $\gamma_{\mathrm{ME}}$ is determined from the minimum virtuality imposed by the QEDPS evolution. If there is no 'Radiative Return' the contribution of this second configuration is negligible small. However, for the process $e^{+} e^{-} \rightarrow \nu \bar{\nu} \gamma(\gamma)$ above the $Z^{0}$ threshold its contribution to the total cross section becomes sizeable, typically about a few $\%$.

Besides the above-mentioned pure QED corrections another class of electroweak higher order corrections should be included in the calculations. The grc $\nu \nu \gamma$ package has two schemes to do this: The running coupling constant and the $G_{\mu}$ scheme [16. In the former the coupling constant of the fermionfermion- $Z$ vertex, $g_{f f Z}$, is determined by evolving it from zero momentum transfer to the mass squared of the $\nu \bar{\nu}$ system, $q_{Z}^{2}$, which differs from one event to another. It varies according to the renormalization group equation (RGE) as

$$
g_{f f Z}\left(q_{Z}^{2}\right)=g_{f f Z}(0)\left(1-\frac{\alpha}{3 \pi} \sum_{i} C_{i} e_{i}^{2} \log \frac{q_{Z}^{2}}{m_{i}^{2}}\right)^{-1},
$$

where $\alpha=1 / 137.036$ is the QED coupling at the zero momentum transfer, $C_{i}$ the color factor, $e_{i}$ the electric charge, $m_{i}$ the mass of the $i$ 's fermion, and $i$ runs over all massive fermions. In this scheme $g_{f f Z}\left(q_{Z}^{2}\right)$ should be fixed event-by-event, but is common for all $Z$-exchange diagrams in the same event.

The latter scheme is such that the weak couplings are determined through the weak-mixing angle, $\sin \theta_{W}$, which is given by

$$
\sin ^{2} \theta_{W}=\frac{\pi \alpha\left(q^{2}\right)}{\sqrt{2} G_{\mu} M_{W}^{2}} \frac{1}{1-\Delta r},
$$

where $M_{W}$ being the $W$-boson mass and $G_{\mu}$ the muon decay constant. In this scheme the input is the precisely measured value of $G_{\mu}$ at zero momentum transfer and $\alpha\left(q^{2}\right)$ is the QED coupling evolved to a typical energy scale of the processes which should be chosen by the users. In the present version of the program the loop corrections expressed by $\Delta r$ in the formula is ignored $(\Delta r=$ $0)$. Then the difference between the running $g_{f f Z}$ scheme and the $G_{\mu}$ scheme is to use the fixed energy scale(for $G_{\mu}$ ) or the event-dependent energy scale(for running $\left.g_{f f Z}\right)$.

The package grc $\nu \nu \gamma$ has a switch to choose either of the above corrections or no higher-order corrections. When users select the last option, the weak couplings are simply fixed by $M_{W}$ and $M_{Z}$ through the on-shell relation, $\sin ^{2} \theta_{W}=1-\frac{M_{W}^{2}}{M_{Z}^{2}}$, where $M_{Z}$ is the mass of the $Z$-boson. 


\section{$3 \quad$ Numerical Results}

\section{$3.11 \gamma$ test}

The numerical results for the single-photon emission are given in this section. All the three generators have been run and compared. The parameters used in the calculations are summarized in Table 1.

\begin{tabular}{|c|c||c|c|}
\hline$M_{Z}$ & $91.187 \mathrm{GeV}$ & $\Gamma_{Z}$ & $2.49 \mathrm{GeV}$ \\
\hline$M_{W}$ & $80.22 \mathrm{GeV}$ & $m_{e}$ & $0.511 \times 10^{-3} \mathrm{GeV}$ \\
\hline$\alpha(0)$ & $1 / 137.04$ & $\alpha\left(M_{W}^{2}\right)$ & $1 / 128.07$ \\
\hline
\end{tabular}

Table 1: Parameters used in the calculations.

The weak coupling constant is determined scheme by scheme according to the selection of the higher-order corrections as explained in the end of the previous section. The visible photon is defined by Eq.(1) with $E_{0}=1 \mathrm{GeV}$ and $\theta_{0}=10^{\circ}$.

First the results for $\nu_{\mu}$ are discussed. In this case four program packages are available: the analytic $\mathcal{O}(\alpha)$ calculations, [ ] NUNUGPV, the present one and KORALZ. The last one also uses the complete matrix-elements for this process including the YFS exponentiation. The cross sections from four independent generators are visualized in Fig.3, and the numbers at four energy points are shown in Table 2. In these calculations $\alpha=1 / 137.04$ and $\sin ^{2} \theta_{W}=$ $1-M_{W}^{2} / M_{Z}^{2}$ are commonly used for all four packages. The statistical errors to the numbers in the table are around $0.2 \%$ due to the numerical integration. The results from KORALZ are very close to those from the $\mathcal{O}(\alpha)$ calculation. Since the former includes the soft-photon exponentiation but the latter does not, one sees that the resultant difference in the total cross sections is less than $1 \%$. In the grc $\nu \nu \gamma$ package, however, QEDPS allows also the hard-photon exponentiation in the LL approximation. From the table this brings an effect of around $1.4 \%$. By comparing three Monte Carlo program packages one can conclude that the theoretical uncertainty from the ISR corrections is about $1 \%$. The results of NUNUGPV are lower than grc $\nu \nu \gamma$ by around $2 \%$.

\footnotetext{
${ }^{2}$ The formulae given in Ref.[5] are used in this paper.
} 


\begin{tabular}{|c|c|c|c|c|}
\hline & $160 \mathrm{GeV}$ & $170 \mathrm{GeV}$ & $180 \mathrm{GeV}$ & $190 \mathrm{GeV}$ \\
\hline grc $\nu \nu \gamma$ & 2.470 & 2.033 & 1.709 & 1.460 \\
\hline $\mathcal{O}(\alpha)$ corr. & 2.436 & 2.006 & 1.689 & 1.449 \\
\hline KORALZ & 2.437 & 2.009 & 1.697 & 1.459 \\
\hline NUNUGPV & 2.410 & 1.987 & 1.668 & 1.430 \\
\hline
\end{tabular}

Table 2: Total cross sections of single-photon emission for the $\nu_{\mu}$ process in $p b$. The on-shell scheme without any higher-order electro-weak corrections is used for all packages.

The differential cross sections with respect to the CMS energy of the pair $\nu \bar{\nu}$, its longitudinal component, energy and transverse energy of the hard photon are compared in Fig.4. The shape of the distributions from these three packages ( $\operatorname{grc} \nu \nu \gamma, \mathcal{O}(\alpha)$, and KORALZ) is in good agreement. A more detailed comparison between grc $\nu \nu \gamma$ and KORALZ on the same distributions is given in Fig.5, which shows the ratio of the results. Besides the overall factors these two are consistent.

The effects of the higher-order corrections due to the running $f f Z$ coupling are shown in Fig. 6 and Table 3 at four energy-points. The higher order

\begin{tabular}{|l|c|c|c|c|}
\hline & $160 \mathrm{GeV}$ & $170 \mathrm{GeV}$ & $180 \mathrm{GeV}$ & $190 \mathrm{GeV}$ \\
\hline grc $\nu \nu \gamma\left(\nu_{\mu}\right.$ only $)$ & 2.470 & 2.033 & 1.709 & 1.469 \\
\hline$\uparrow+$ running $g_{f f Z}$ & 2.846 & 2.334 & 1.968 & 1.678 \\
\hline$\uparrow$ for all neutrino species & 11.16 & 9.70 & 8.62 & 7.85 \\
\hline
\end{tabular}

Table 3: Total cross sections of single-photon emission for the $\nu_{\mu}$ and all neutrino processes using grc $\nu \nu \gamma$ in $p b$.

effects from the running $g_{f f Z}$ contribute to the total cross section by around $13 \%$. After summing up all the neutrino species the total cross sections are also summarized in the figure and the table. The contribution from the $W$-exchange diagrams(last three diagrams in Fig.1) amounts about $25 \%$.

The scheme dependence of the higher-order corrections is found by grc $\nu \nu \gamma$ to be around $1 \%$ as seen from Fig. 7 and Table 4 . Here $q^{2}=M_{W}^{2}$ is chosen as the energy scale for the $G_{\mu}$ scheme. The discrepancy between two schemes 


\begin{tabular}{|l|c|c|c|c|}
\hline & $160 \mathrm{GeV}$ & $170 \mathrm{GeV}$ & $180 \mathrm{GeV}$ & $190 \mathrm{GeV}$ \\
\hline grc $\nu \nu \gamma+$ running $g_{f f Z}$ & 11.16 & 9.70 & 8.62 & 7.85 \\
\hline grc $\nu \nu \gamma+G_{\mu}$ scheme & 10.98 & 9.56 & 8.53 & 7.81 \\
\hline NUNUGPV & 10.76 & 9.41 & 8.37 & 7.69 \\
\hline
\end{tabular}

Table 4: Total cross sections of the single-photon emission for the all neutrino processes using grc $\nu \nu \gamma$ and NUNUGPV in $p b$. The latter package implements the $G_{\mu}$ scheme for the higher-order corrections.

can be understood as follows. Two different energy scales are involved in this process: The colliding energy of the initial $e^{+} e^{-}$and the $Z$-boson mass. [1 The contributions to the cross section from these two energy regions are compatible in magnitude as seen from Fig.4. An expected difference in the total cross section could be estimated as $1-\alpha^{2}\left(M_{Z}^{2}\right) / \alpha^{2}\left(E_{\mathrm{LEP} 2}^{2}\right) \approx 2 \%$ between these two scales. Hence the $G_{\mu}$ scheme which depends on a single fixed energy scale is not suitable for those processes that involve different energy scales.

The differential distributions in $G_{\mu}$ scheme obtained by grc $\nu \nu \gamma$ are in good agreement with those by NUNUGPV as shown in Fig.8.

\section{$3.22 \gamma$ test}

For the double-photon emission a similar comparison is also made between grc $\nu \nu \gamma$ and NUNUGPV. Both packages equip the exact matrix-elements for the double-photon emission associated with all neutrinos including $\nu_{e} \bar{\nu}_{e}$ (Fig.2) and the $G_{\mu}$ scheme for the higher order corrections but different ways for the LL exponentiation for ISR, QEDPS or $p_{T}$-depending structure function. Hence a difference occurs again in the treatment of the second configuration of the photon emission discussed in section 2. In applying grc $\nu \nu \gamma$ to the double-photon emission, one has to take into account the possibility that one of the two photons supplied by the matrix-elements can be invisible(in $\Omega_{\mathrm{i}}$ ) and one photon from QEDPS becomes visible. The configuration in which both photons from the matrix-elements disappear into $\Omega_{\mathrm{i}}$ is ignored safely. The

\footnotetext{
${ }^{3}$ There is another energy scale $q^{2}=0$ for the real photon emission. This can be easily separated by using a fixed value of $\alpha=1 / 137.04$ at the $e-e-\gamma$ vertex in the matrix elements and the ISR part.
} 
total cross sections of the double-photon emission are summarized in Fig.9 and Table 5, where a slight difference is seen between two packages. The

\begin{tabular}{|l|c|c|c|c|}
\hline & $160 \mathrm{GeV}$ & $170 \mathrm{GeV}$ & $180 \mathrm{GeV}$ & $190 \mathrm{GeV}$ \\
\hline grc $\nu \nu \gamma$ & 0.687 & 0.599 & 0.537 & 0.481 \\
\hline NUNUGPV & 0.669 & 0.578 & 0.507 & 0.472 \\
\hline
\end{tabular}

Table 5: Total cross sections of the double-photon emission for all neutrino processes using grc $\nu \nu \gamma$ and NUNUGPV in $p b$. The latter package implements the $G_{\mu}$ scheme for the higher-order corrections.

differential distributions are in good agreement with each other as shown in Fig.10.

\section{Anomalous $W W \gamma$ Coupling}

In the grc $\nu \nu \gamma$ package the anomalous coupling of the $W-W-\gamma$ vertex is prepared. The program includes only those terms which conserve $C$ and $P$ invariance, derived from the following effective Lagrangian 17]:

$$
\begin{array}{r}
L_{e f f}=-i e\left[\left(1+\Delta g_{1 \gamma}\right)\left(W_{\mu \nu}^{\dagger} W^{\mu}-W^{\dagger \mu} W_{\mu \nu}\right) A^{\nu}+\right. \\
\left(1+\Delta \kappa_{\gamma}\right) W_{\mu}^{\dagger} W_{\nu} A^{\mu \nu} \\
\left.+\frac{\lambda_{\gamma}}{M_{W}^{2}} W_{\lambda \mu}^{\dagger} W_{\nu}^{\mu} A^{\lambda \nu}\right]
\end{array}
$$

where $W_{\mu \nu}=\partial_{\mu} W_{\nu}-\partial_{\nu} W_{\mu}, A_{\mu \nu}=\partial_{\mu} A_{\nu}-\partial_{\nu} A_{\mu}$. Here $\Delta g_{1 \gamma}, \Delta \kappa_{\gamma}$ and $\lambda_{\gamma}$ stand for the anomalous coupling parameters which vanish in the standard model.

To find the region sensitive to the anomalous TGC in the photon phasespace, the energy and angular distributions are examined at $\Delta g_{1 \gamma}=0, \Delta \kappa_{\gamma}=$ -10 and $\lambda_{\gamma}=0$. This is shown in Fig.11. The sensitive region, as expected from a glance at the diagrams, locates in the high energy side and large angle region, except for around the 'Radiative Return', where the annihilation dominates the process. Then the experimental cuts of $20 \mathrm{GeV}<E_{\gamma}<55$ $\mathrm{GeV}$ or $65 \mathrm{GeV}<E_{\gamma}$ and $45^{\circ}<\theta_{\gamma}<135^{\circ}$ are effective to enhance the signal from the anomalous TGC. The total cross section as a function of $\Delta \kappa_{\gamma}$ and $\lambda_{\gamma}$ with the experimental cuts at the CMS energy at $170 \mathrm{GeV}$ is shown in Fig.12. 
Once $200 p b^{-1}$ of the accumulated luminosity and $100 \%$ of the acceptance are assumed, $128 \pm 11$ events will be observed. The error given here is only a statistical one. The sensitivity of -9 to +6 for $\lambda_{\gamma}$ and -3 to +10 for $\Delta \kappa_{\gamma}$ is expected at the three-sigma limit if one supposes only the statistical error of the measurements. Since the higher order corrections also change the total cross sections under the above cuts, as pointed out in Ref.[6], a careful investigation is needed on the systematics from those corrections.

\section{Conclusions}

An event generator grc $\nu \nu \gamma$ for the processes $e^{+} e^{-} \rightarrow \nu \bar{\nu} \gamma$ and $e^{+} e^{-} \rightarrow$ $\nu \bar{\nu} \gamma \gamma$ has been proposed. The exact matrix elements for single- and doublephoton emission are prepared by GRACE. QEDPS is used as a tool for the ISR corrections. For the $\nu_{\mu}$ case the total cross sections and the hard-photon distributions of grc $\nu \nu \gamma$ are compared with those from the $\mathcal{O}(\alpha)$ calculation, KORALZ and NUNUGPV. It is found that the theoretical uncertainty for the ISR corrections is under control at the $1 \%$ level. The systematics of the $G_{\mu}$ scheme from the double energy scales involved in the reaction is estimated to be around 1\%. The energy spectrum of the hard-photons is in a reasonable agreement with independent calculations from KORALZ and NUNUGPV up to the double-photon emission.

Concerning $\nu_{e}$ a similar comparison with NUNUGPV has been done, though in lacking $\mathcal{O}(\alpha)$ calculations. With this process there is some opportunity to measure the anomalous TGC at the $W-W-\gamma$ vertex which appears in the single energetic events at the LEP-2 when the luminosity is enough accumulated. Since only the experimental observable is the total cross section, a careful investigation of the systematic errors must be done.

The authors would like to thank G. Montagna, O. Nicrosini, and F. Piccinini for the various discussions on the comparison between grc $\nu \nu \gamma$ and NUNUGVP, and S. Jadach for KORALZ. We also wish to thank D. Perret-Gallix at LAPP, and K. Tobimatsu and K. Kato at Kogakuin-University for their fruitful discussions and suggestions.

This work was supported in part by the Ministry of Education, Science and Culture, Japan under the Grant-in-Aid for Scientific Research 
No.11440083.

\section{References}

[1] ALEPH Collab., D. Buskulic et al., Phys. Lett. B313 (1993) 520, DELPHI Collab., P. Abreu et al., Z.Phys. C74 (1997) 577, L3 Collab., M. Acciarri et al., Phys. Lett. B431 (1998) 199, OPAL Collab., R. Akers et al., Z.Phys. C65 (1995) 47.

[2] ALEPH Collab., R. Barate et al., Phys. Lett. B429 (1998) 201, ALEPH Collab., R. Barate et al., CERN-EP/98-053, DELPHI Collab., P. Abreu et al., Phys. Lett. B380 (1996) 471, DELPHI Collab., P. Abreu et al., CERN-EP/98-142, L3 Collab., M. Acciarri et al., Phys. Lett. B415 (1997) 299, L3 Collab., M. Acciarri et al., CERN-EP/98-150, OPAL Collab., K.Ackerstaff et al., Eur. Phys. J. C2 (1998) 607, OPAL Collab., K.Ackerstaff et al., CERN-EP/98-143.

[3] F.A. Berends, et at., Nucl. Phys. B301 (1988) 583.

[4] F.A. Berends, G.F. Burgers W.L. van Neerven, Phys. Lett. B177 (1986) 191 , H. Veltman, Nucl. Phys. B312 (1989) 1.

[5] M. Igarashi, N. Nakazawa, Nucl. Phys. B288 (1987) 301.

[6] A. Jachołkowska, J. Kalinowski, Z. Wa̧s, CERN-TH/98-055,hepph/9803375,

'KoralZ v4.0', S. Jadach, B. Ward, Z. Wạs. Comp. Phys. Comm. 79 (1994) 503.

[7] S. Jadach, B.F.L Ward, Z. Wạs, Phys. Lett B257 (1991) 213, see also references in [6].

[8] G. Montagna, M. Moretti, O. Nicrosini, F. Piccinini Nucl. Phys. B541 (1999) 31, Acta Phys.Polon. B29 (1998) 2699.

[9] F. Caravaglios, M. Moretti, Phys. Lett. B358(1995) 332. 
[10] O. Nicrosini and L. Trentadue, Nucl. Phys. B318 (1989) 1, O. Nicrosini and L. Trentadue, hys. Lett. B231 (1989) 487.

[11] T. Ishikawa, T. Kaneko, K. Kato, S. Kawabata, Y. Shimizu, H. Tanaka. GRACE manual, KEK report 92-19, 1993.

[12] T. Munehisa, J. Fujimoto, Y. Kurihara, Y. Shimizu, Prog. Theor. Phys. 95 (1996) 375.

[13] Y. Kurihara, J. Fujimoto, T. Munehisa, Y. Shimizu, Prog. Theor. Phys. 96 (1996) 1223.

[14] R. Odorico, Nucl. Phys. B172 (1980) 157, G. Marchesnin, B.R. Webber, Nucl. Phys. B238 (1984) 1.

[15] S. Kawabata, Comp. Phys. Comm. 41 (1986) 127; ibid., 88 (1995) 309.

[16] 'WW cross-sections and distributions', W. Beenakker et al. in 'Physics at LEP2', eds. G. Altarelli, T. Sjöstrand, F. Zwirner, CERN 96-01, (1996), Vol.1, p.79.

[17] K. Hagiwara, R.D. Peccei and D. Zeppenfeld, Nucl. Phys. B282 (1987) 253. 


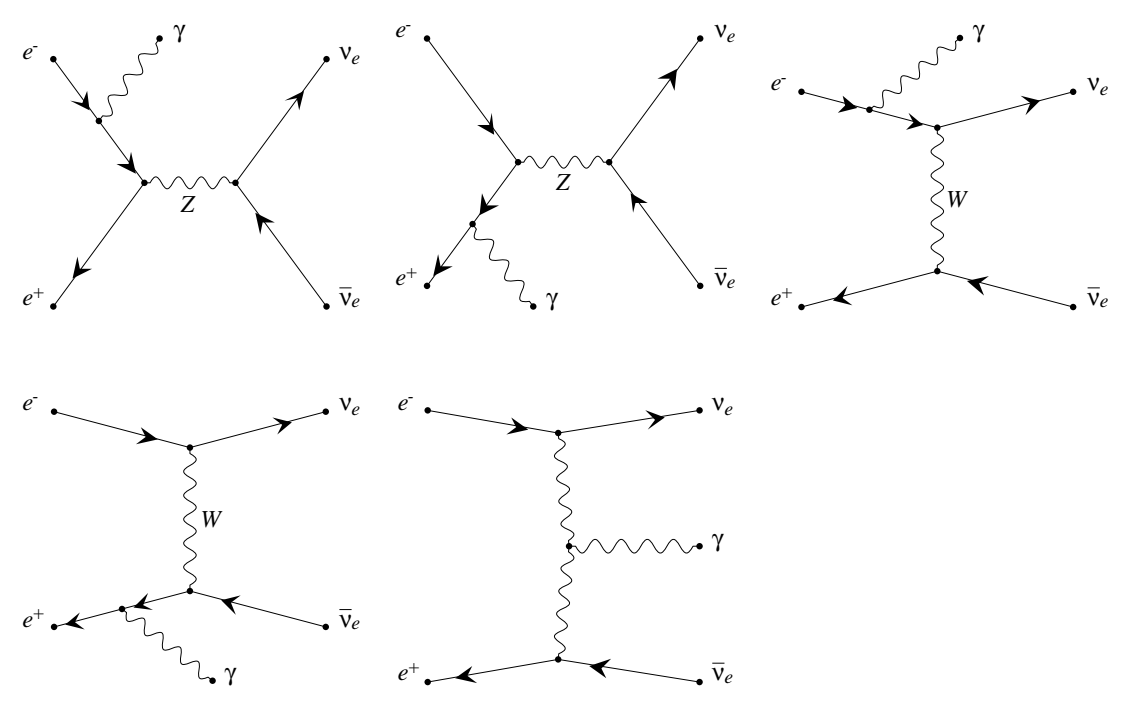

Figure 1: Feynman diagrams of the process $e^{+} e^{-} \rightarrow \nu_{e} \bar{\nu}_{e} \gamma$. Only the first two diagrams appear in $\nu_{\mu}$ and $\nu_{\tau}$ neutrino production. 

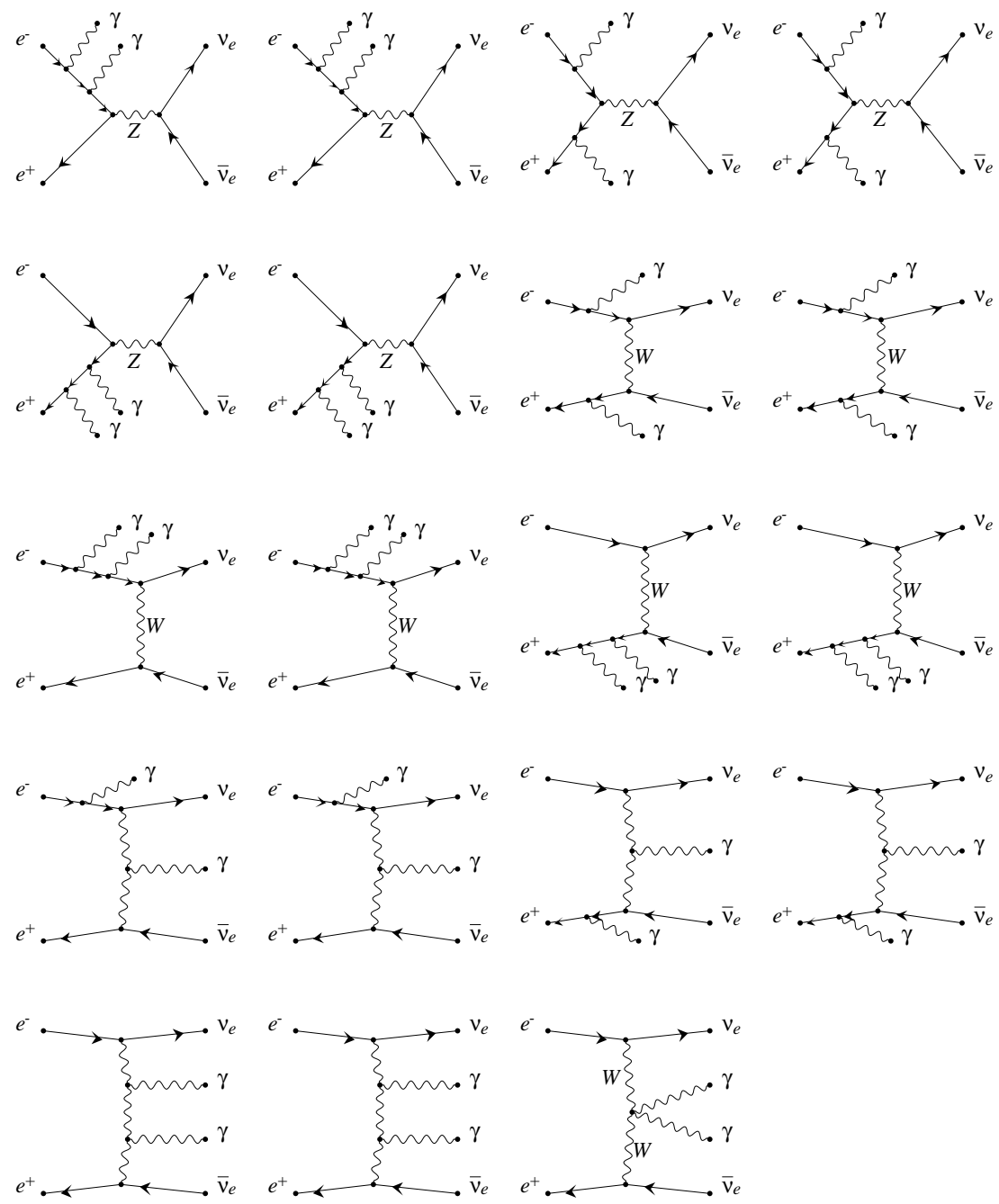

Figure 2: Feynman diagrams of the process $e^{+} e^{-} \rightarrow \nu_{e} \bar{\nu}_{e} \gamma \gamma$. Only the first six diagrams appear in $\nu_{\mu}$ and $\nu_{\tau}$ neutrino production. 


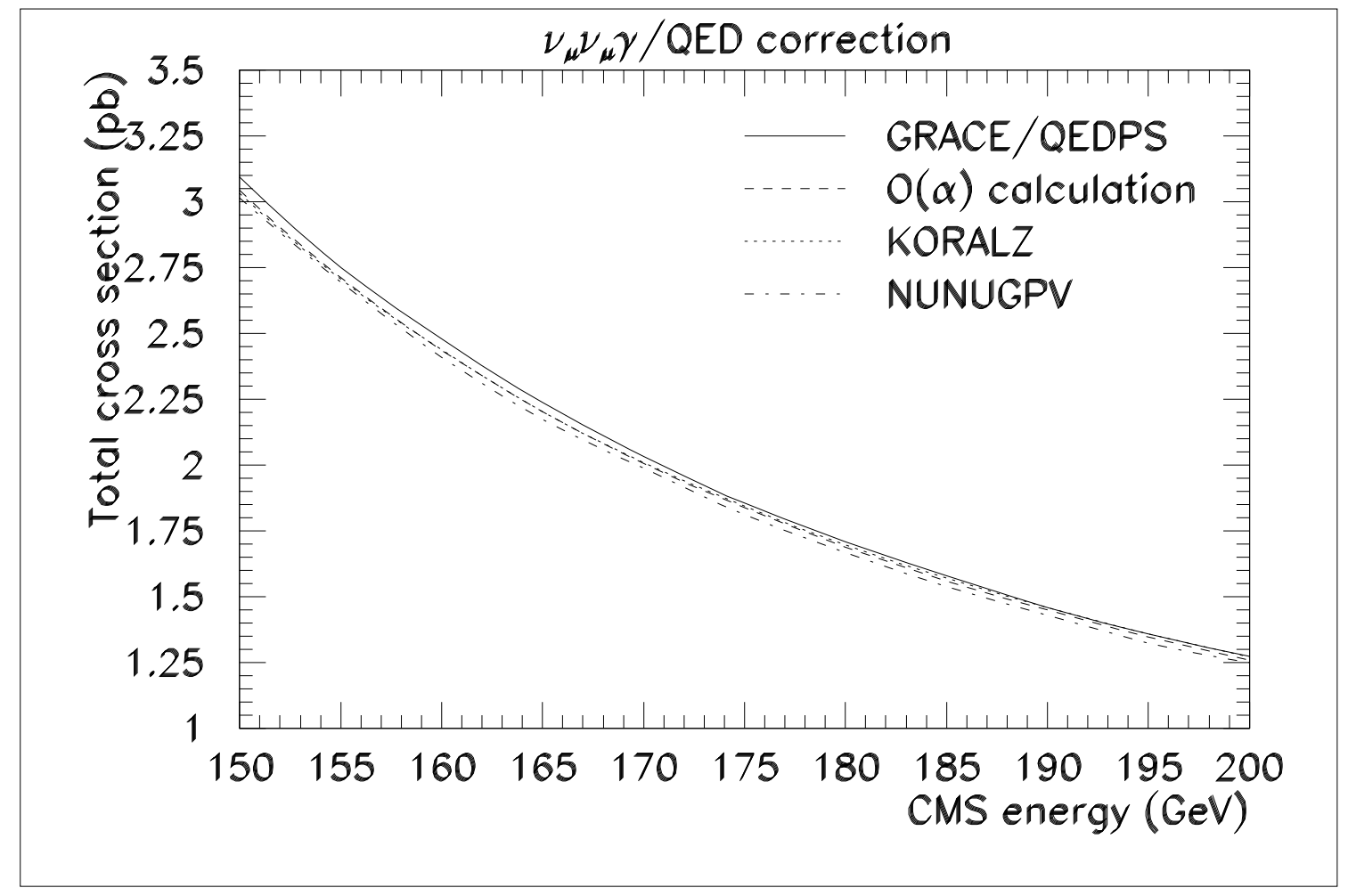

Figure 3: Total cross sections of the single-photon emission for the $\nu_{\mu}$ process in $p b$. The on-shell scheme without any higher-order electro-weak correction is used for all the program packages. 


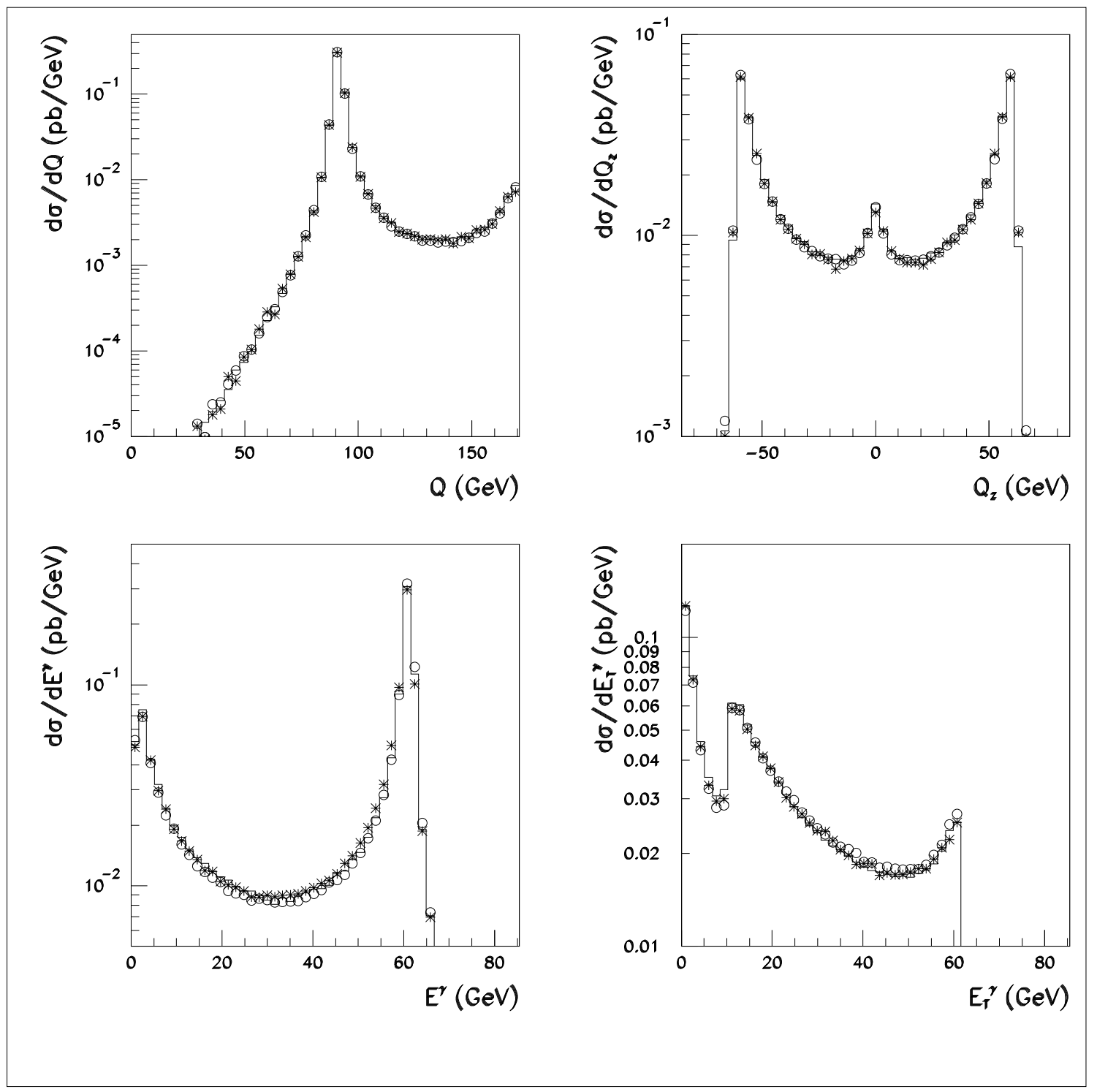

Figure 4: Differential cross sections with respect to the CMS energy of $\nu \bar{\nu}$ system, its longitudinal component, energy and transverse energy of the hard photon obtained by grc $\nu \nu \gamma$ (solid histograms), $\mathcal{O}(\alpha)$ calculations (stars), and KORALZ (circles) at the CMS energy of $170 \mathrm{GeV}$. 


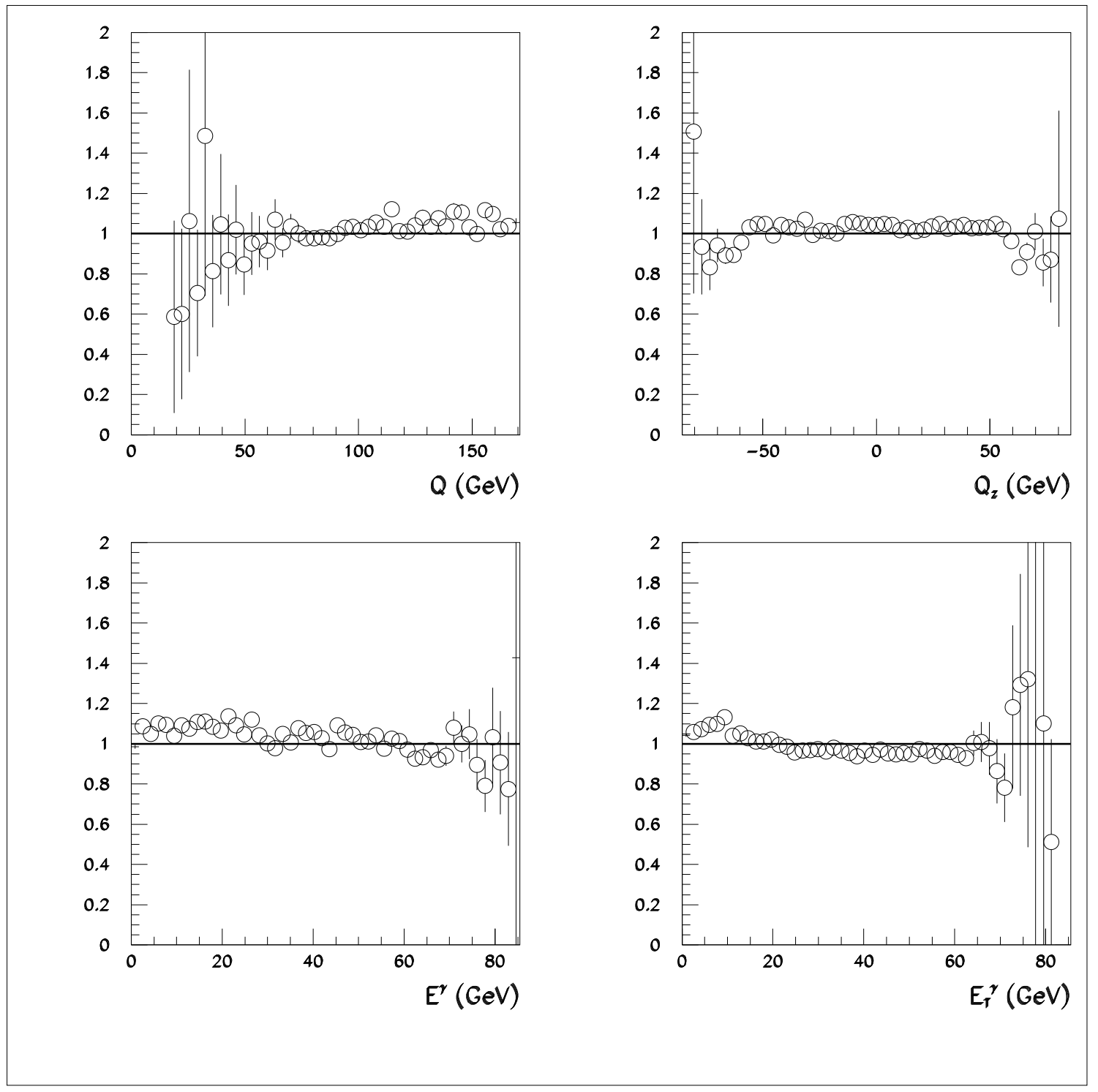

Figure 5: Ratio between grc $\nu \nu \gamma$ and KORALZ for the same distributions as in Fig.4. The error bars show the statistical errors of the numerical integration. 


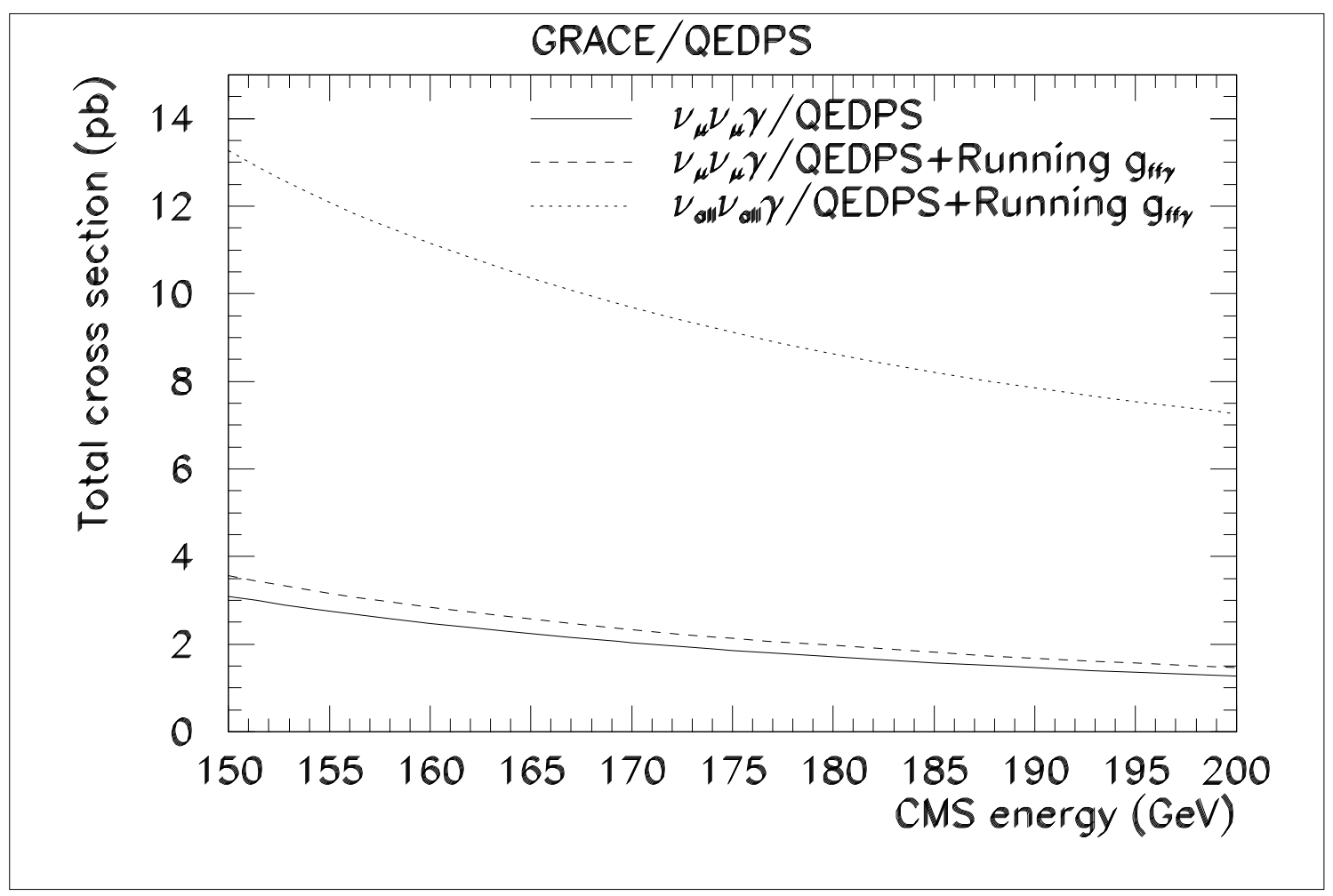

Figure 6: Total cross sections of the single-photon emission for the $\nu_{\mu}$ and all neutrino processes using grc $\nu \nu \gamma$ in $p b$. 


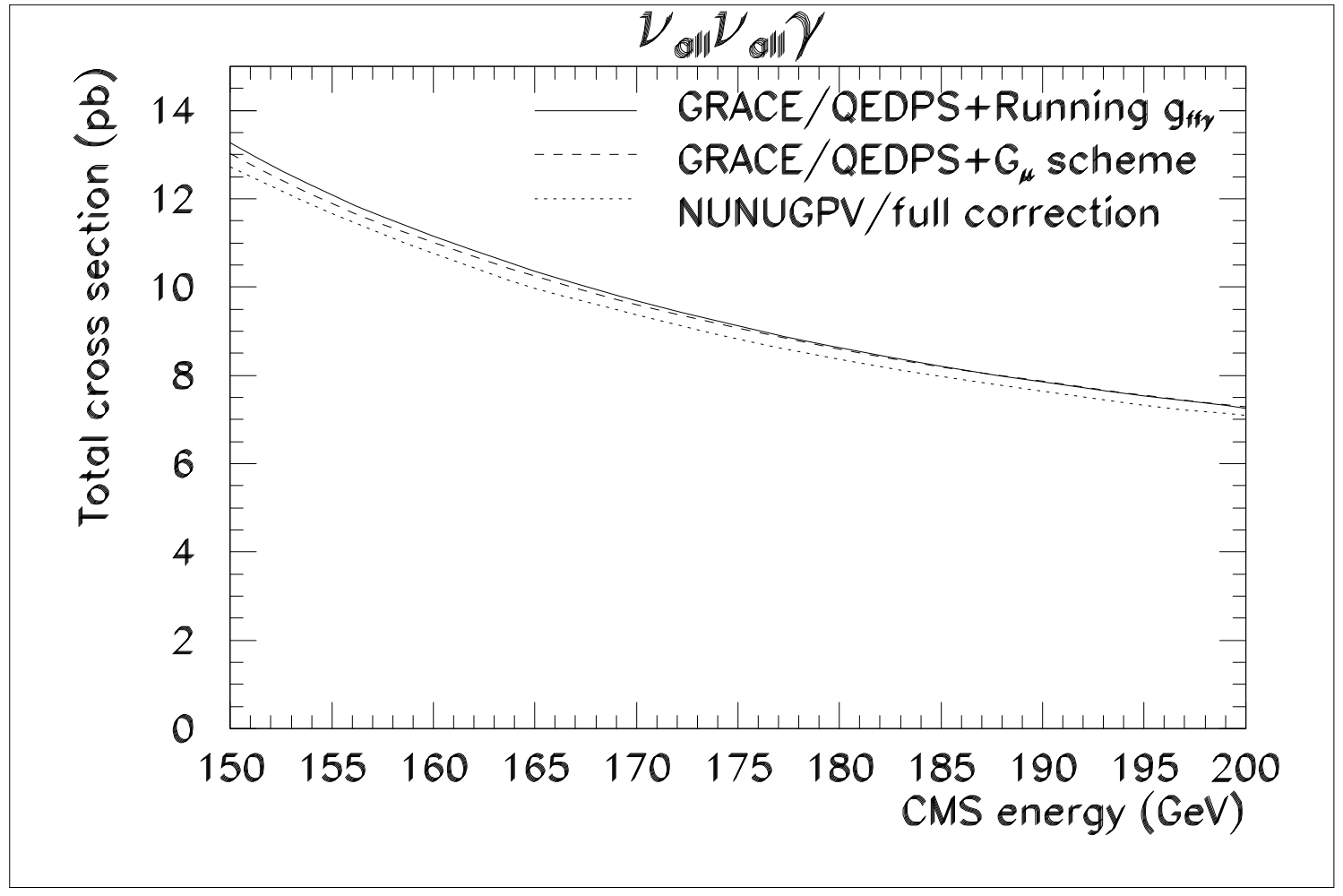

Figure 7: Total cross sections of the single-photon emission for all the neutrino processes using grc $\nu \nu \gamma$ and NUNUGPV in $p b$. The latter implements the $G_{\mu}$ scheme for the higher order corrections. 


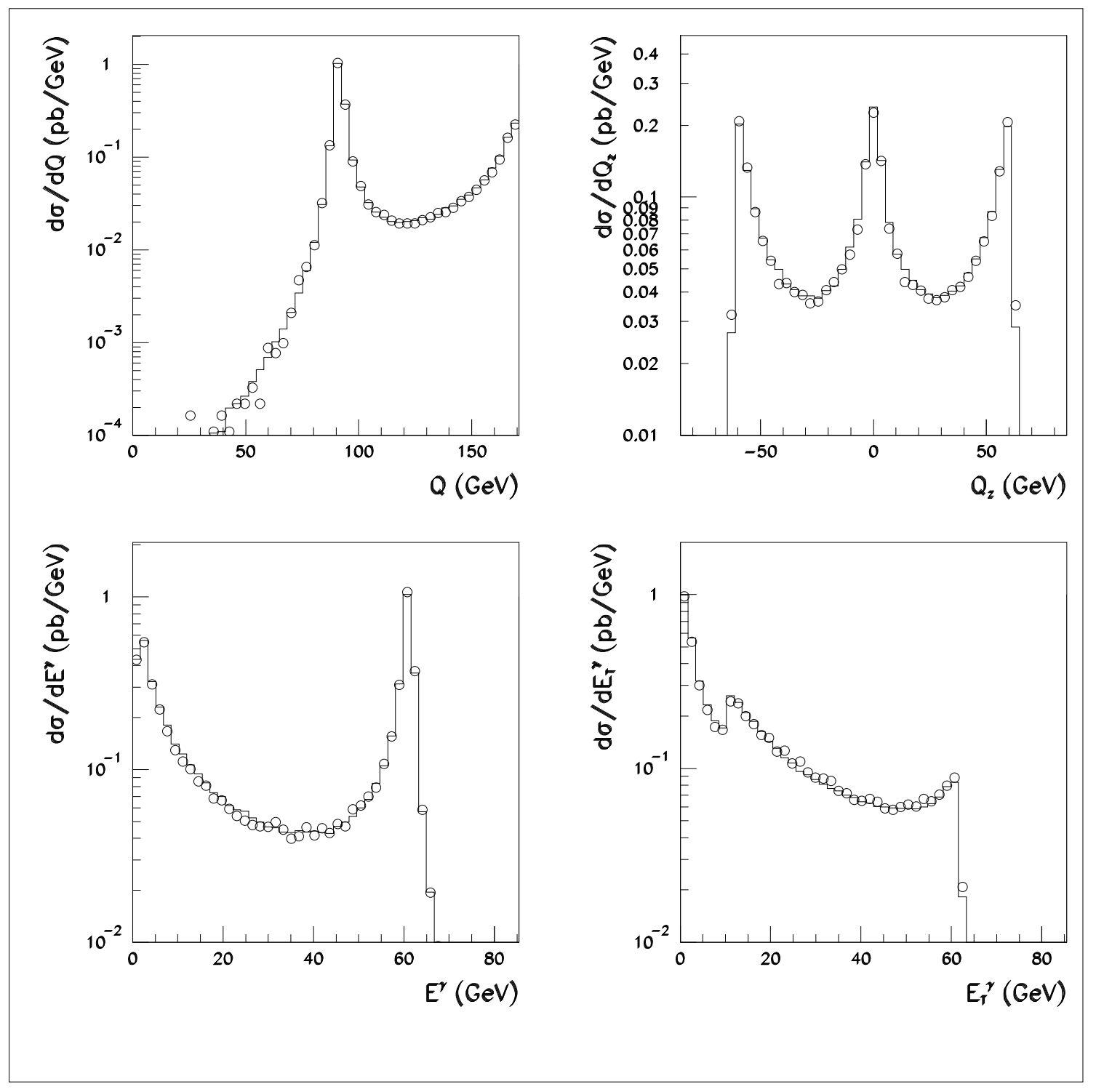

Figure 8: Differential cross sections with respect to the CMS energy of the $\nu \bar{\nu}$ system, its longitudinal component, energy and transverse energy of the hard photon obtained by grc $\nu \nu \gamma$ (solid histograms) and NUNUGPV (circles) at the CMS energy of $170 \mathrm{GeV}$. 


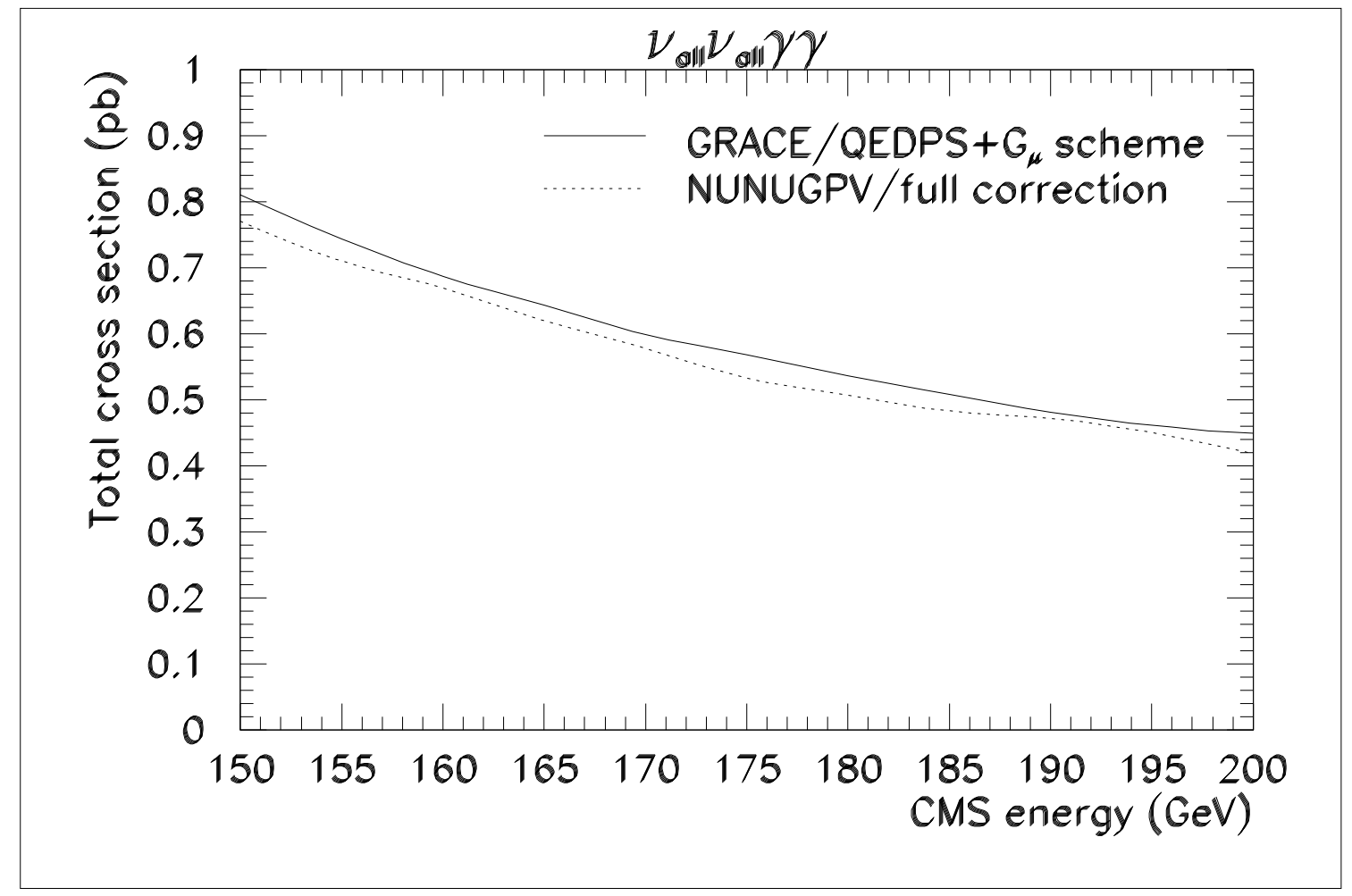

Figure 9: Total cross sections of the double-photon emission for all the neutrino processes using grc $\nu \nu \gamma$ and NUNUGPV in $p b$. The latter implements the $G_{\mu}$ scheme for the higher order corrections. 


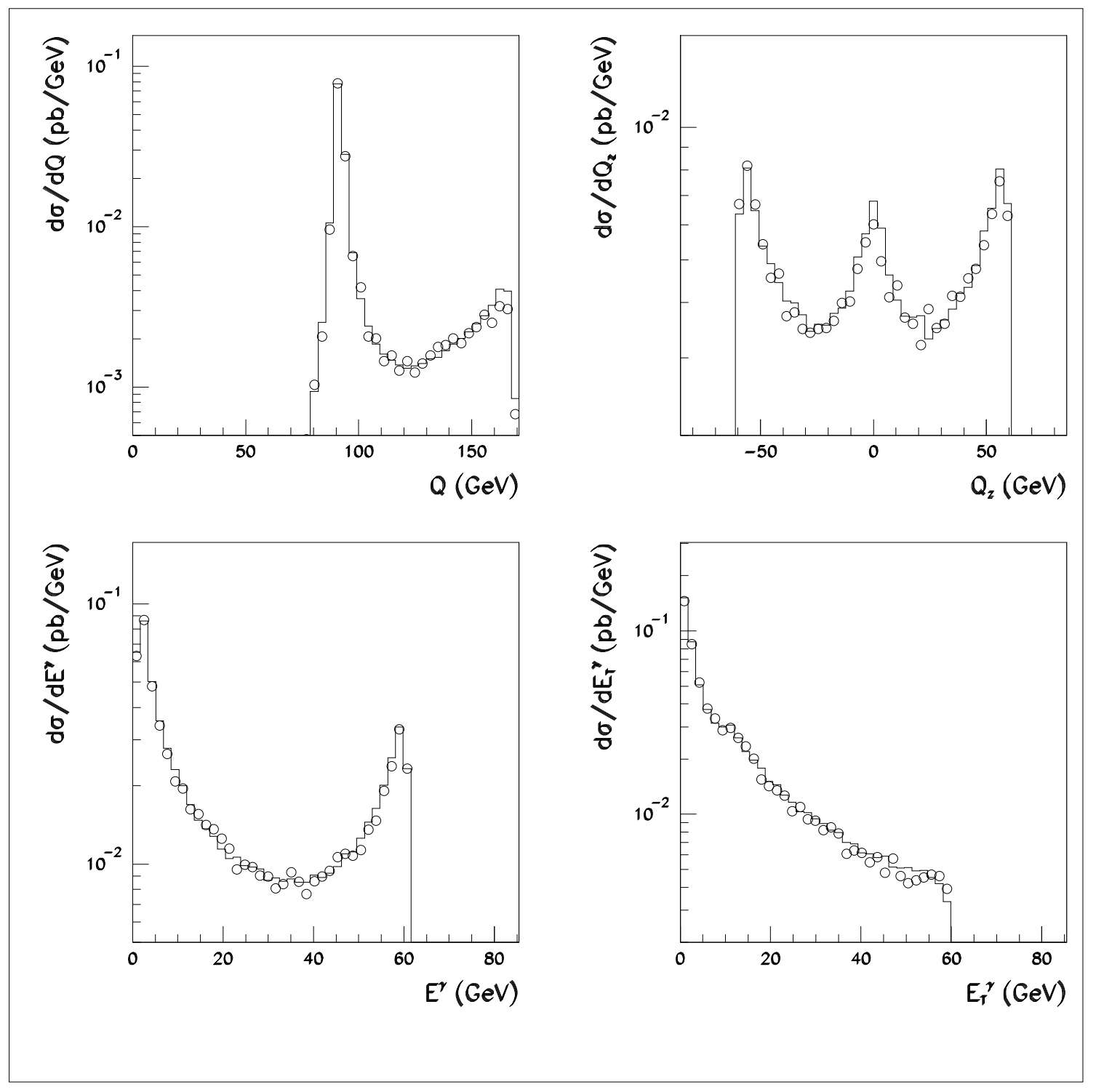

Figure 10: Differential cross sections with respect to the CMS energy of the $\nu \bar{\nu}$ system, its longitudinal component, energy and transverse energy of the hard photon obtained by grc $\nu \nu \gamma$ (solid histograms) and NUNUGPV (circles) at the CMS energy of $170 \mathrm{GeV}$. 


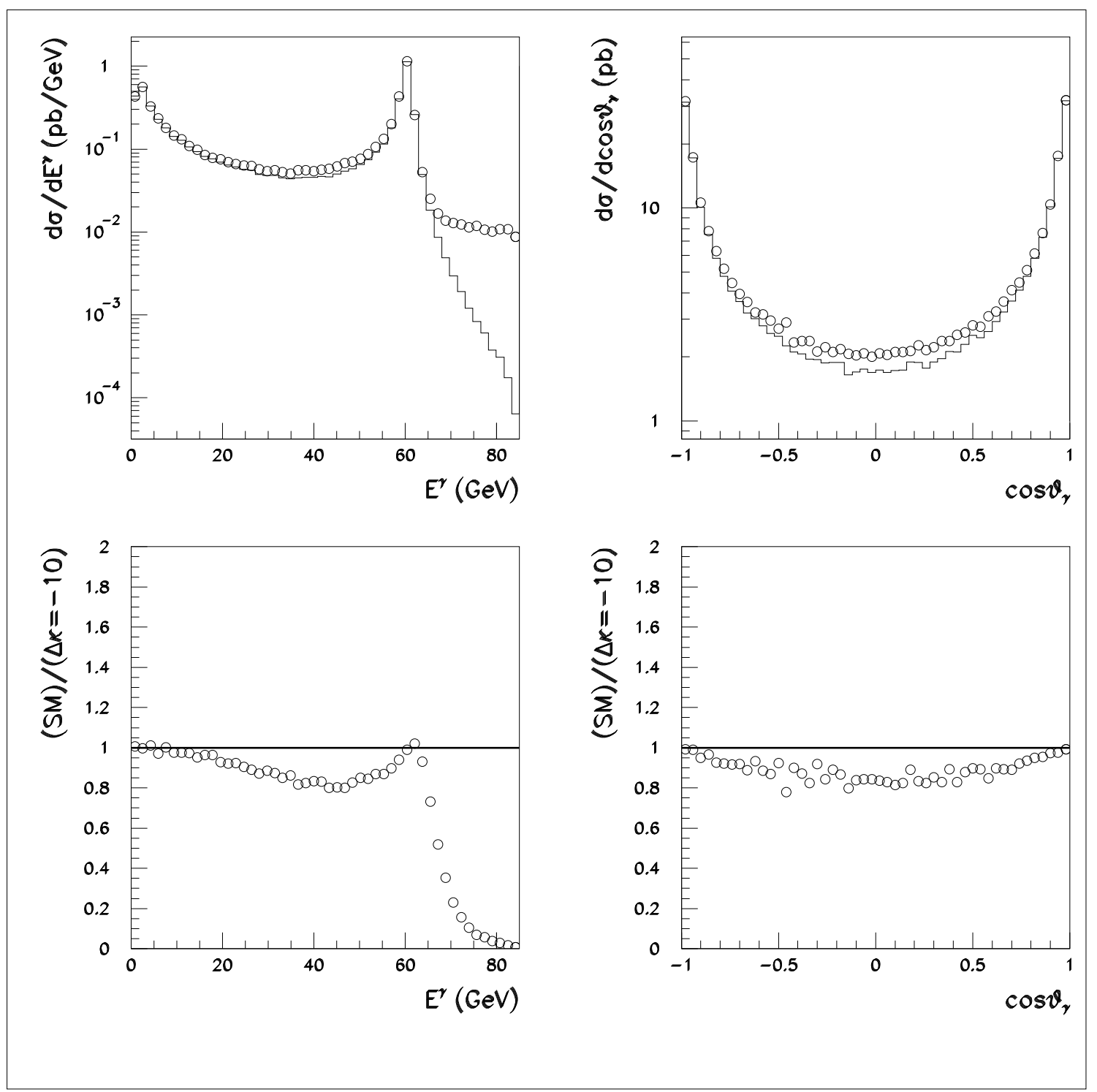

Figure 11: Energy and angular distributions of the hard photon obtained by grc $\nu \nu \gamma$ with the standard TGC (solid histograms) and the anomalous TGC with $\Delta k_{\gamma}=-10$ at the CMS energy of $170 \mathrm{GeV}$. The distributions of the ratio between the standard and the anomalous TGC are also shown. 


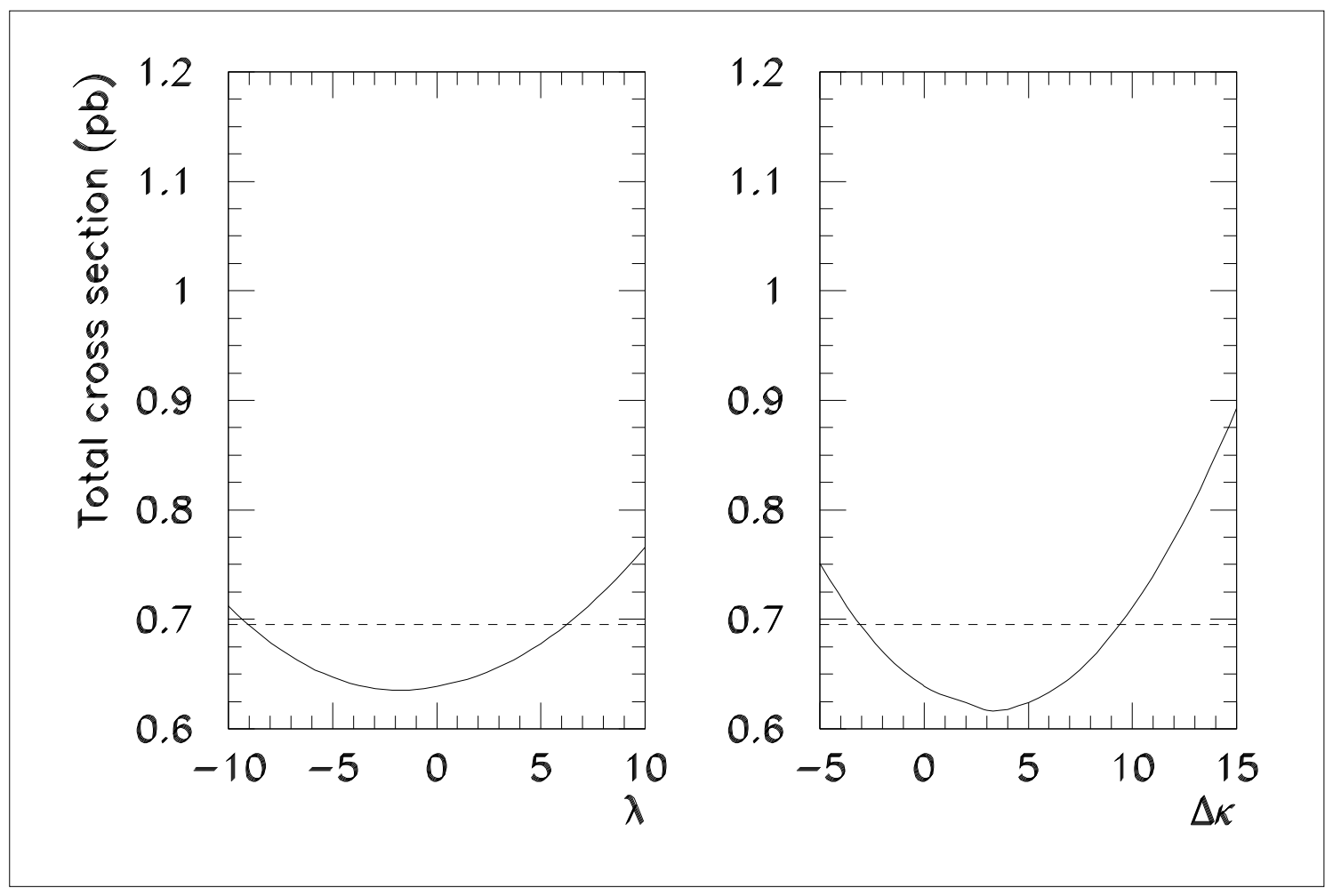

Figure 12: Total cross sections of the single hard-photon events as a function of $\lambda_{\gamma}$ (left) and $\Delta \kappa_{\gamma}$ (right) at the CMS energy of $170 \mathrm{GeV}$. The dashed lines are the expected experimental upper limit at three standard-deviations of the statistical error from the standard model parameters after $200 p b^{-1}$ data accumulation. 


\section{Appendix}

\section{A.1 How to install the program}

The source code is available by anonymous ftp from ftp.kek.jp in the directory kek/minami/grcnna. The grc $\nu \nu \gamma$ system contains the complete set of FORTRAN sources for $e^{+} e^{-} \rightarrow \nu \bar{\nu} \gamma$ and $e^{+} e^{-} \rightarrow \nu \bar{\nu} \gamma \gamma$ matrix-elements and the four libraries, i.e., BASES/SPRING, CHANEL, QEDPS and utilities for kinematics. Those source codes are written in FORTRAN77. grc $\nu \nu \gamma$ has been developed on HP-UX, but should run on any UNIX platform with a FORTRAN complier.

The procedure of installation is as follows:

1. Editing Makefile.

The following macros in Makefile should be taken care of by users themselves. For example, GRCNNADIR defines the directory name where grc $\nu \nu \gamma$ is installed. The values of FC and FOPT define the relevant compiler name and option for your system. The other macros can be left as they are.

$$
\begin{aligned}
& \text { GRCNNADIR }=\text { directory where grc } \nu \nu \gamma \text { are installed. } \\
& \text { LIBDIR }=\text { directory where libraries are installed. } \\
& \text { (default is } \$ \text { (GRCNNADIR)/lib.) } \\
& \text { BINDIR = directory where an executable is installed. } \\
& \text { (default is } \$ \text { (GRCNNADIR)/bin.) } \\
& \text { MACHINE }=\text { [hpux } \mid \text { hiux } \mid \text { sgi } \mid \text { dec } \mid \text { sun }] \\
& \text { FC } \quad=\text { FORTRAN compiler command name. } \\
& \text { FOPT } \quad=\text { FORTRAN compiler options. } \\
& \text { CERNLIBS }=\text { CERNLIB including the jetset library. } \\
& \text { For HP-UX, -L/cern/pro/lib -ljetset74 } \\
& \text {-lpacklib -lkernlib -L/lib/pa1.1/ -lm }
\end{aligned}
$$

2. Compilation.

By executing command make install the executable of the interface program is generated at BINDIR. Furthermore four libraries, BASES/SPRING, CHANEL, QEDPS and kinematics utility library, are generated in LIBDIR. 


\section{A.2 How to run the program}

According to the number of hard-photons, two directories are prepared: $\$$ (GRCNNADIR)/1a and \$(GRCNNADIR)/2a. Three FORTRAN files and a Makefile are stored in each directory.

For the numerical integration, BASES:

- mainbs.f: A main program of the numerical integration.

- usrprm.f: A subroutine for parameter setting.

For the event generation, SPRING:

- mainsp.f: A main program of the event generation. Event-loop is also included.

The subroutine, usrprm.f, is used commonly for both of BASES and SPRING.

Users can set the parameters of the calculations by editing the file usrprm.f. The key words are as follows;

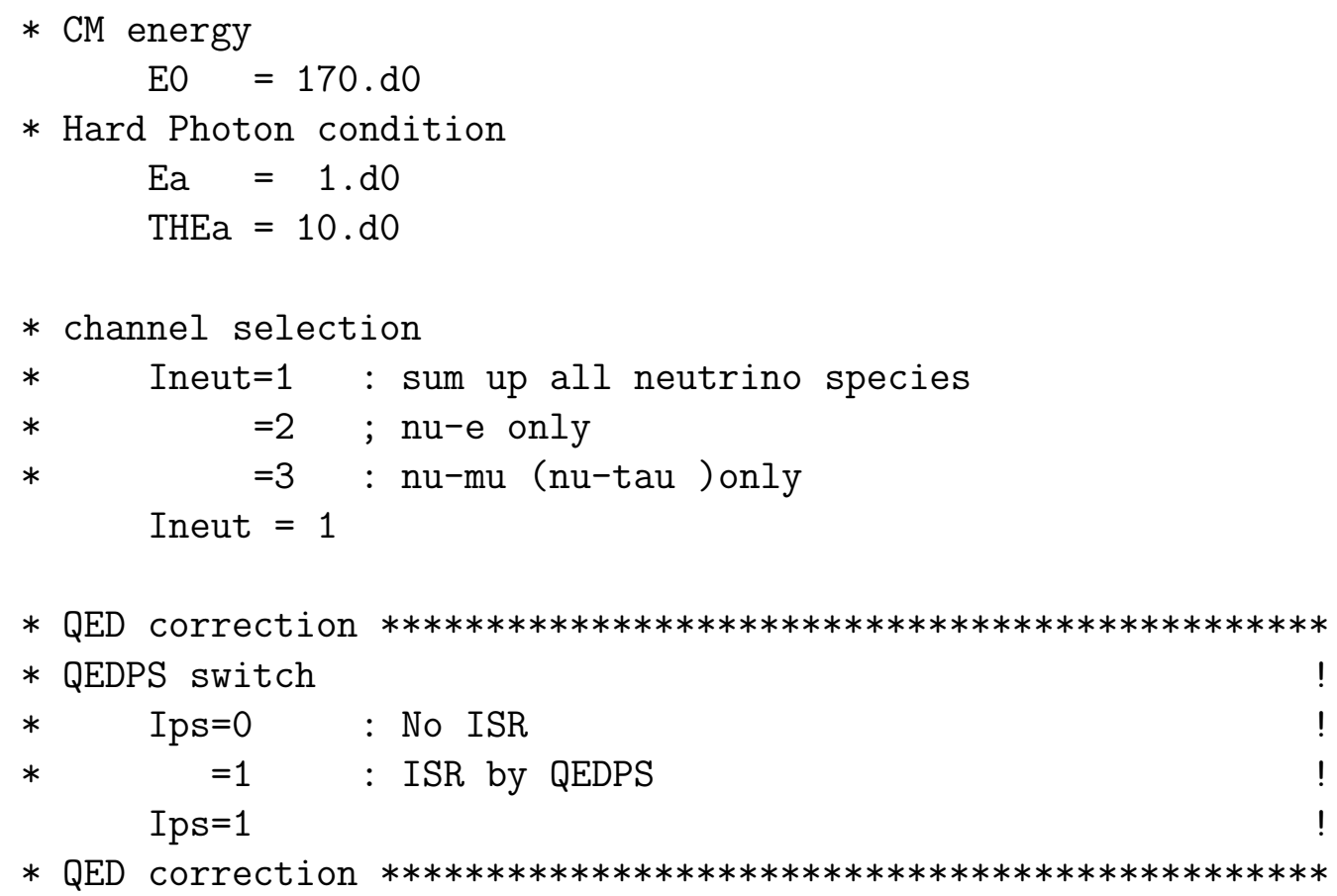




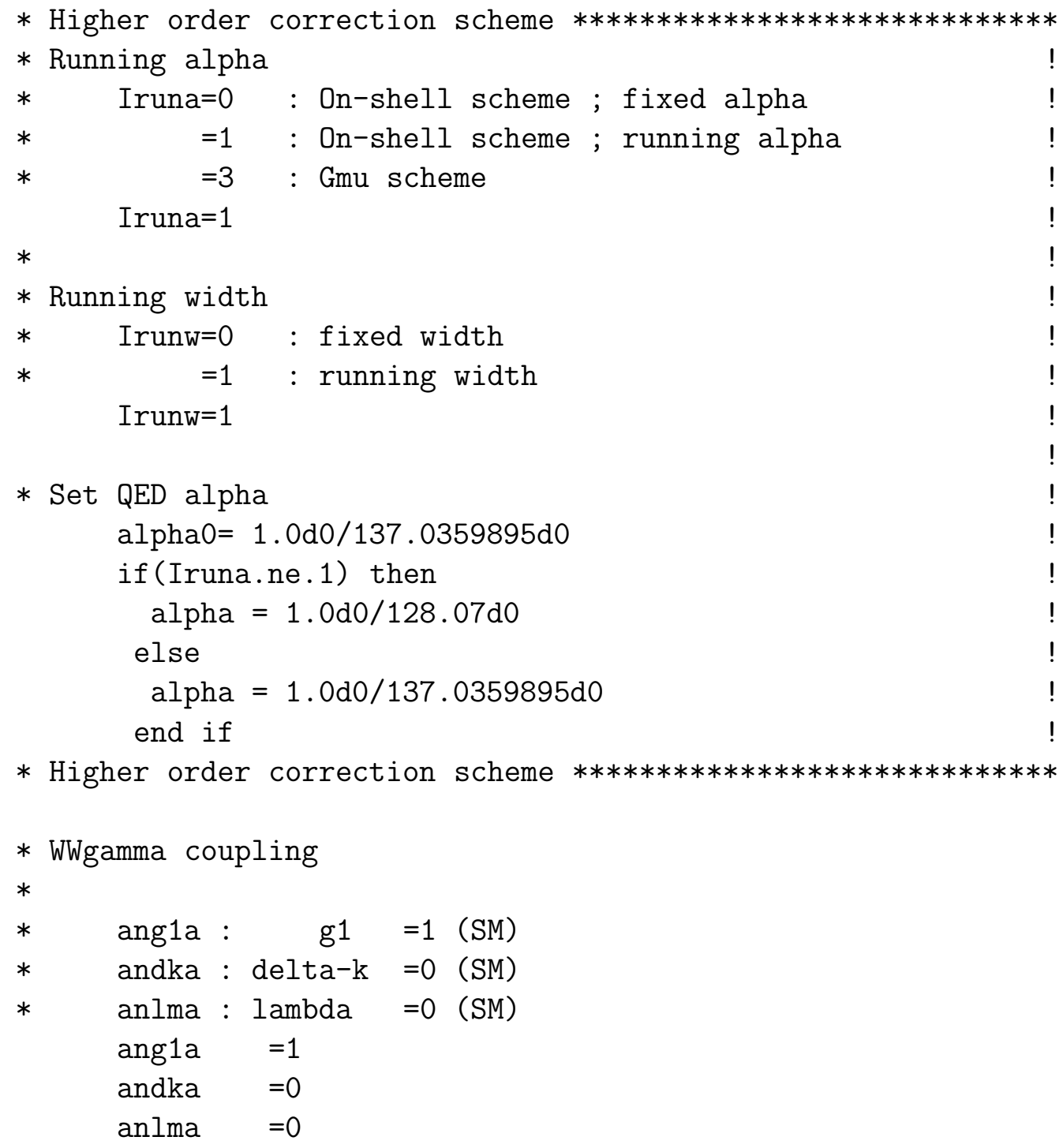

The parameters for the anomalous TGC are available only for the singlephoton case.

Users can proceed with the calculations as follows:

i) Change directory by typing

$\%$ cd $\$($ GRCNNADIR) $/ 1 \mathrm{a}$ 
or

$\% \operatorname{cd} \$$ (GRCNNADIR) $/ 2 \mathrm{a}$

ii) Edit usrprm.f to set user parameters.

iii) Create an executable integ for the integration by typing

$\%$ make integ

iv) Numerical integration is actually performed by typing

$\% \quad$ integ

The results of the integration step are displayed on the console as well as written in an output file bases.result. The total cross section in $p b$ and the estimated statistical error are shown on the last line, under Cumulative Result, in the table of the Convergence Behavior for the Integration step. The differential cross sections are also printed as a function of the energy, scattering angle of each particle and invariant masses of any two final particles. The probability distribution of the integrand is written in a file bases. data which will be used in the event generation step by spring.

v) Before running the event generation, users may edit mainsp. $f$ to set additional parameters if needed and call the user's own analysis routines.

The following is the structure of the generated mainsp.f, where fourmomentum of all particles including soft-photons generated by QESPS are stored in the common/lujets/ in the JETSET format when subprogram sp2lnd is called in the event-loop:

Program mainsp

implicit $r e a l * 8(a-h, o-z)$

external func

real $* 4 \quad \mathrm{p}, \mathrm{v}$ 


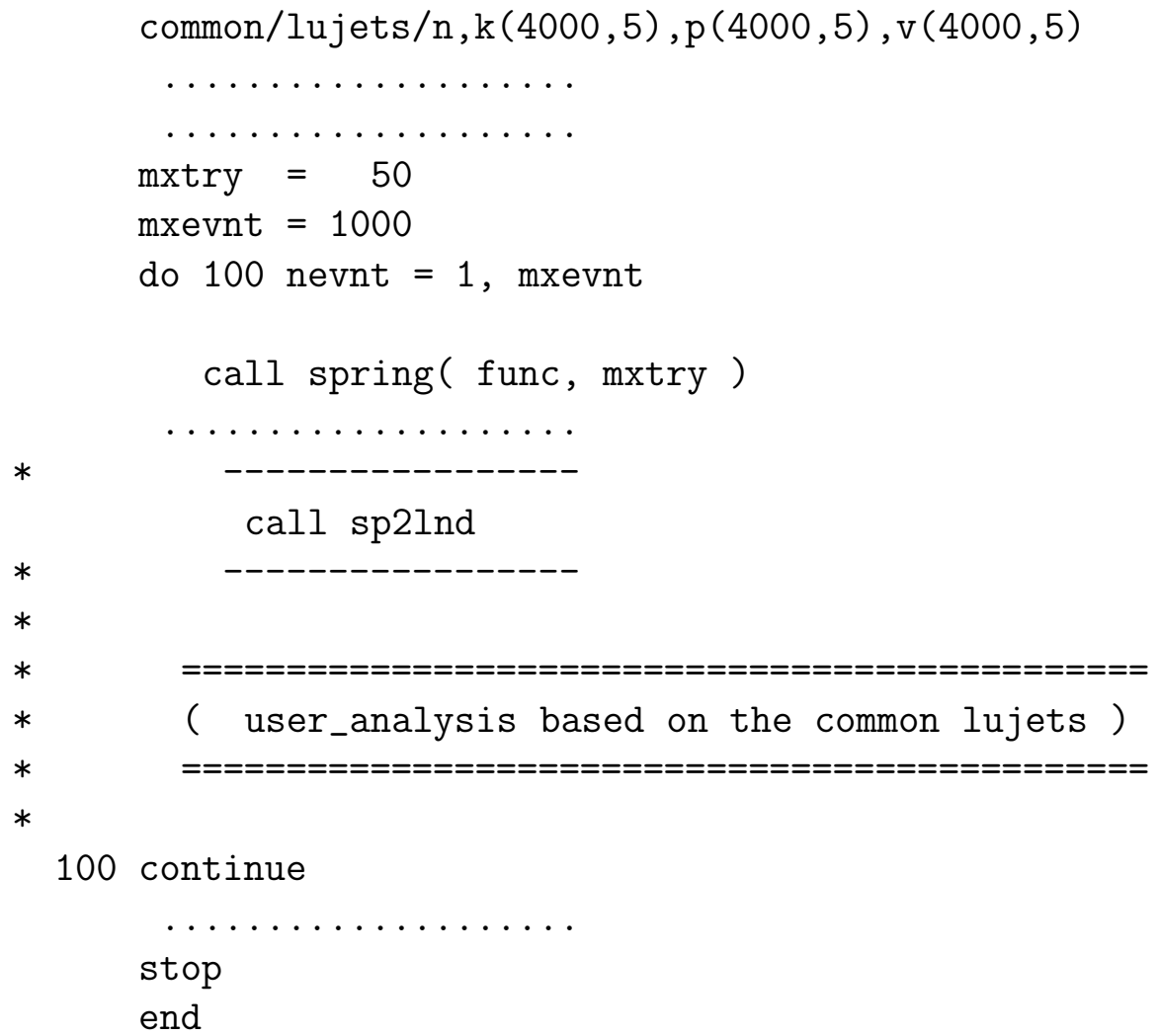

iv) Create an executable spring for event generation by typing

$\%$ make spring

vii) Start the event generation by typing

$\%$ spring

Information concerning the event generation will be written in the spring.result file. Users should pay attention to the histograms generated in this step. The distributions of the generated events are superimposed with the character " 0 " on the histograms generated in the integration step. These two distributions should be consistent with each other within the statistical error of the generation. For the details of the output files of BASES and SPRING, users can consult Ref. [15]. 